\title{
RETOMADA DA COLHEITA MECANIZADA EM CANA-DE-AÇÚCAR PÓS-CHUVA E SUA RELAÇÃO COM ATRIBUTOS FÍSICOS DO SOLO
}

\section{Claudinei Midena Midena1, Juliano Carlos de Souza1 ${ }^{1}$, Jackeline Matos Nascimento $^{1 *}$, Mateus Luiz Secretti $^{1}$, Sálvio Napoleão Soares Arcoverde ${ }^{2}$}

${ }^{1}$ Centro Universitário da Grande Dourados, Dourados, 79824-900, Mato Grosso do Sul, Brasil

${ }^{2}$ Universidade Federal da Grande Dourados, Dourados, 79825-070, Mato Grosso do Sul, Brasil

*Autor para correspondência: Jackeline Matos Nascimento; e-mail: jackeline_ms@yahoo.com.br

Recebido: 07/10/2020, Aceito: 2408/2021, Publicado: 18/10/2021

\begin{abstract}
Resumo
A compactação é uma das principais causas de degradação estrutural em solos cultivados com cana-de-açúcar, devido, principalmente, à elevada carga das máquinas utilizadas na colheita, bem como a realização desta operação com alto teor de umidade do solo. Com isso, o objetivo dessa pesquisa foi avaliar a retomada da colheita mecanizada em cana-de-açúcar pós-chuva nas propriedades físicas de um Latossolo Vermelho Escuro distrófico, textura média. $\mathrm{O}$ experimento foi implantado após uma chuva de $30 \mathrm{~mm}$ na área experimental, onde os tratamentos foram compostos pela interação de três fatores, sendo eles: umidade (colheita pós-chuva em 1, 2, 3 e 4 dias), ocasião de coleta de solo (antes e depois da colheita) e profundidade de coleta $(0-0,10 ; 0,10-0,20$ e 0,20 $0,30 \mathrm{~m}$ ), para determinação da densidade e porosidade total. Em todos os dias avaliados, o tráfego de máquinas na colheita de cana-de-açúcar ocasiona maior compactação na camada superficial de solo; e quando este tráfego de máquinas é realizado no quarto dia pós-chuva, cuja umidade média do solo foi de aproximadamente de 7\%, ocorre menor densidade e maior porosidade total. A densidade do solo mostrou-se um indicador sensível à compactação como consequência do tráfego de máquinas para retomada de colheita de cana-de-açúcar.
\end{abstract}

Palavras-chave: compactação, densidade do solo, mecanização agrícola, porosidade total, umidade do solo.

\section{RESUMPTION OF MECHANIZED HARVESTING IN AFTER-RAIN SUGARCANE AND ITS RELATIONSHIP WITH SOIL PHYSICAL ATTRIBUTES}

\begin{abstract}
Compaction is one of the main causes of structural degradation in soils cultivated with sugarcane, mainly due to the high weight of the harvesting machines, as well as the performance of this operation with high soil moisture content. The objective was to evaluate the resumption of mechanized harvesting in post-rain sugarcane on the physical properties of a medium texture dystrophic Red Latosol. The experiment was implemented after a $30 \mathrm{~mm}$ rain in the experimental area, where the treatments were composed by the interaction of three factors, namely: moisture (post-rain harvest in 1, 2, 3 and 4 days), occasion of soil collection (before and after harvest) and collection depth (0-0.10, 0.10-0.20 and 0.20-0.30 m), to determine the density and total porosity. On all evaluated days, the traffic of machines for cane harvesting causes greater compaction in the superficial layer of soil; and when this machine traffic is carried out on the fourth post-rain day, whose average soil moisture was approximately $7 \%$, there is less density and greater total porosity. Soil density proved to be a sensitive indicator of compaction as a consequence of the traffic of machines for resuming cane harvesting.
\end{abstract}

Keywords: agricultural machinery, compaction, moisture, soil porosity, soil bulk density 


\section{Introdução}

A cana-de-açúcar é uma das culturas de maior importância no agronegócio brasileiro, visto que apresenta elevado potencial para diversos usos na agroindústria e na cogeração de energia elétrica. $\mathrm{O}$ aumento da demanda por produtos derivados da canade-açúcar é responsável pelo crescimento do setor sucroenergético brasileiro, o que resulta na expansão dos canaviais e investimentos na construção de novas usinas, sobretudo na região Centro-sul (Arcoverde et al., 2019a).

O sistema de produção de cana-de-açúcar requer mecanização intensiva, resultando em mudanças nas propriedades físicas do solo e processos relacionados. $\mathrm{O}$ preparo do solo é usado para incorporar corretivos e fertilizantes quando a cana é estabelecida pela primeira vez, e novamente em intervalos de aproximadamente cinco anos quando a produção de cana-de-açúcar começa a diminuir e é replantada. Com a recente mudança para a colheita mecanizada, o tráfego intensivo de máquinas pode estar contribuindo para a degradação física do solo nessas áreas (Cherubin et al., 2016).

As sucessivas operações mecanizadas - do preparo do solo até a colheita, resultam em problemas de degradação do solo, sendo o principal a compactação (GARCIA et al., 2016). Sobretudo, devido ao tráfego de máquinas em conteúdos inadequados de água no solo (Severiano et al., 2008), que pode afetar a produção e a longevidade do canavial (Guimarães Júnnyor et al., 2019), bem como restringir a área explorada pelo sistema radicular (Souza et al., 2019).

Assim, o intenso tráfego de máquinas de colhedora e do transbordo usados na colheita sem queima e mecanizada tem afetado as propriedades físicas do solo, com diminuição da porosidade e consequente aumento da densidade do solo, o que resulta também no aumento da resistência à penetração de raízes, diminuição da aeração e do escoamento superficial, favorecendo a formação de processos erosivos (Cherubin et al., 2016). Desse modo, a colheita mecanizada de cana-de-açúcar quando feita em solos com elevados teores de água, tem sido a principal e maior causadora da compactação dos solos nos sistemas produtivos da cultura. Consequentemente, se torna imprescindível determinar a umidade do solo antes de iniciar qualquer operação que envolva maquinários agrícolas que possam possibilitar a compactação do mesmo (Severiano et al., 2008).

Portanto, o objetivo desse estudo foi avaliar a influência da retomada da colheita mecanizada em cana-de-açúcar pós-chuva nas propriedades físicas de um Latossolo Vermelho Distrófico.

\section{Material e Métodos}

\section{Caracterização da área de estudo}

$\mathrm{O}$ experimento foi conduzido no ano agrícola 2018/2019, em uma área com o total de 8,52 ha, localizada na fazenda Bocaiúva, no município de Caarapó, situado no sul da região Centro Oeste do Brasil, no sudoeste de Mato Grosso do Sul, tendo como coordenadas geográficas aproximadas de latitude $22^{\circ} 38^{\prime} 46^{\prime \prime} \mathrm{S}$ e longitude $54^{\circ} 45^{\prime} 32^{\prime \prime}$ W Greenwich, altitude média 455 metros. O clima da região é do tipo Cwa (Clima subtropical de inverno seco), temperado e úmido com inverno seco, e precipitação média anual de $1500 \mathrm{~mm}$ (FIETZ et al., 2017), e temperatura média anual de $22^{\circ} \mathrm{C}$, segundo a classificação de Köppen. $\mathrm{O}$ solo é classificado como Latossolo Vermelho Distrófico argilúvico álico A moderado (PRADO, 2013), com textura franco-arenoso, tendo $16 \%$ de argila, $68 \%$ de areia e $16 \%$ de Silte, baseado na classificação proposta pelo Departamento de Agricultura dos Estados Unidos (USDA). A variedade de cana-de-açúcar plantada na área foi SP83-2847.

\section{Equipamentos em colheita mecanizada de cana-de- açúcar}

Os equipamentos agrícolas utilizados para a colheita da cana-de-açúcar foram: colhedora da marca John Deere, modelo CH570, ano de fabricação 2017; e trator da marca John Deere, modelo 6180J, ano de fabricação 2017, sendo este acoplado um transbordo agrícola de arrasto da marca Civemasa, modelo Tandem com capacidade total de $17 \mathrm{Mg}$.

\section{Coleta de dados}

Os tratamentos do estudo foram o resultado da combinação de três fatores: umidade (colheita póschuva em 1, 2, 3 e 4 dias), ocasião de coleta de solo (antes e depois da colheita) e profundidade de coleta ( 0 $0,10,0,10-0,20$ e $0,20-0,30 \mathrm{~m})$. A colheita da cana-deaçúcar foi realizada em ciclo de cana-planta, por isso antes da colheita mecanizada considerou-se $\mathrm{o}$ tratamento "sem tráfego de equipamentos" (STE), enquanto, após a colheita mecanizada, em seus diferentes dias pós-chuva, considerou-se o tratamento "com tráfego de equipamentos" (CTE).

Em cada tratamento, foram realizadas três repetições na entrelinha de cana, representadas por parcelas de $14 \mathrm{~m} \times 1,5 \mathrm{~m}$. Em cada parcela, foram abertas três trincheiras, para a retirada de amostras de solo com estrutura preservada, nas profundidades de 0 $0,10 \mathrm{~m}, 0,10-0,20 \mathrm{~m}$ e $0,20-0,30 \mathrm{~m}$. As amostras foram coletadas durante quatro dias consecutivos após a chuva, com intervalos de 24 horas, respectivamente 24 , 48,72 e 96 horas, após o final da precipitação de $30 \mathrm{~mm}$. 
As determinações da densidade do solo, porosidade total e umidade do solo seguiram a metodologia proposta por Teixeira et al. (2017). Para a determinação da densidade do solo e da porosidade total, foram coletadas amostras de solo com estrutura preservada com anéis volumétricos de $117,38 \mathrm{~cm}^{3}$, em média; para determinação da umidade do solo utilizaram-se amostras com estrutura alterada. A porosidade total do solo foi obtida pela diferença entre a massa do solo saturado e a massa do solo seco em estufa a $110^{\circ} \mathrm{C}$ durante $24 \mathrm{~h}$; a densidade do solo foi calculada pela relação entre a massa seca a $110^{\circ} \mathrm{C}$ durante $24 \mathrm{~h}$ da amostra de solo do anel volumétrico e o volume do mesmo anel.

\section{Análises estatísticas}

$\mathrm{O}$ experimento foi analisado adotando-se o delineamento inteiramente casualizado com repetições, composto em trifatorial com parcelas subsubdivididas no tempo. Os diferentes valores de umidade do solo constituíram o primeiro fator (parcela principal - PP); ocasiões de coleta de solo, o segundo fator (parcela subdividida - PS); e profundidade de coleta, o terceiro fator (parcela subsubdividida no tempo - PSS).

As análises foram realizadas utilizando-se o programa estatístico Sisvar, por meio do qual os dados foram submetidos ao teste de Tukey a $5 \%$ de probabilidade para comparação das médias, bem como à correlação de Pearson.

\section{Resultados e Discussão}

Com tráfego de equipamentos (CTE), e sem tráfego de equipamentos (STE) não houve diferença entre as profundidades, ou seja, tanto na profundidade superficial $(0-0,10 \mathrm{~m})$, quanto nas mais profundas $(0,10-0,20 \mathrm{~m}$ e $0,20-0,30 \mathrm{~m})$, a umidade não sofreu alteração (Tabela 1). Isso evidencia a importância da cobertura do solo que, mesmo em cana-planta, foi capaz de manter continuidade da umidade no perfil do solo. No fator dias, no entanto, uma menor umidade do solo foi detectada quatro dias após a chuva na camada mais superficial do solo $(0-0,10 \mathrm{~m})$. Possivelmente, esse resultado é atribuído a maior exposição da camada superficial do solo, onde houve maior efeito da evapotranspiração no ressecamento dessa camada com diminuição da sua umidade e também na absorção de água pelas raízes das plantas (REINERT et al., 2008), em relação às camadas subjacentes.
Tabela 1: Umidade do solo, dados em porcentagem \%, latossolo na região de Caarapó MS.

\begin{tabular}{ccccccc}
\hline & \multicolumn{6}{c}{ Profundidade (m) } \\
\cline { 2 - 7 } Dias & \multicolumn{2}{c}{$0-0,10$} & \multicolumn{2}{c}{$0,10-0,20$} & \multicolumn{2}{c}{$0,20-0,30$} \\
\hline & CTE & STE & CTE & STE & CTE & STE \\
1 & $8,27 \mathrm{ab}$ & $7,77 \mathrm{ab}$ & $6,39 \mathrm{~b}$ & $8,12 \mathrm{a}$ & $7,01 \mathrm{a}$ & $7,60 \mathrm{a}$ \\
2 & $8,75 \mathrm{a}$ & $8,13 \mathrm{a}$ & $9,67 \mathrm{a}$ & $8,21 \mathrm{a}$ & $9,12 \mathrm{a}$ & $8,78 \mathrm{a}$ \\
3 & $8,12 \mathrm{ab}$ & $8,26 \mathrm{a}$ & $8,64 \mathrm{ab}$ & $8,41 \mathrm{a}$ & $9,18 \mathrm{a}$ & $8,25 \mathrm{a}$ \\
4 & $6,73 \mathrm{~b}$ & $6,33 \mathrm{~b}$ & $6,99 \mathrm{ab}$ & $7,34 \mathrm{a}$ & $7,41 \mathrm{a}$ & $7,61 \mathrm{a}$ \\
\hline
\end{tabular}

CTE: com tráfego de equipamentos, STE: sem tráfego de equipamentos. Letras iguais maiúsculas na linha e minúscula na coluna, não diferem entre si pelo teste Tukey 0,05 .

$\mathrm{Na}$ profundidade de $0-0,10 \mathrm{~m}$, houve um acréscimo na densidade com o tráfego de equipamentos em todos os dias avaliados, e na camada de $(0,10-0,20$ $\mathrm{m})$ de profundidade houve aumento da densidade com o tráfego nos dias 1, 2 e 3 (Tabela 2). A camada $(0,20-$ $0,30 \mathrm{~m}$ ) só sofreu aumento com o tráfego no primeiro dia de avaliação. A menor média de acréscimo da densidade com tráfego dos equipamentos (CTE), em relação aos dados sem tráfego de equipamentos (STE), foi no $4^{\circ}$ dia de avaliação, o que exerceu menor compactação no solo, isso se explica pelo fato de que no $4^{\circ}$ dia, a umidade do solo decresceu, em média, $1,2 \%$ (Tabela 1), em comparação ao dia anterior (Tabela 2).

Tabela 1: Densidade do solo, gramas por $\mathrm{cm}^{3}$, latossolo na região de Caarapó/MS.

\begin{tabular}{ccccccc}
\hline & \multicolumn{6}{c}{ Profundidade (m) } \\
\cline { 2 - 7 } Dias & \multicolumn{2}{c}{$0-0,10$} & \multicolumn{2}{c}{$0,10-0,20$} & \multicolumn{2}{c}{$0,20-0,30$} \\
\hline & CTE & STE & CTE & STE & CTE & STE \\
1 & 1,71 a A & 1,56 a B & 1,75 a A & 1,67 a B & 1,77 a A & 1,68 ab B \\
2 & 1,77 a A & 1,57 a B & 1,79 a A & 1,71 a B & 1,78 a A & 1,78 a A \\
3 & 1,71 a A & 1,50 ab B & 1,74 a A & 1,66 a B & 1,82 a A & 1,76 a A \\
4 & 1,69 a A & 1,43 b B & 1,60 b A & 1,62 a A & 1,63 b A & 1,64 b A \\
\hline
\end{tabular}

CTE: com tráfego de equipamentos, STE: sem tráfego de equipamentos. Letras iguais maiúsculas na linha e minúscula na coluna, não diferem entre si pelo teste Tukey 0,05 .

Como o solo sob cultivo de cana-de-açúcar, canaplanta, encontra-se menos coeso e com menor continuidade, o tráfego cumulativo de máquinas utilizadas nas operações de manejo ocasionam alteração da estrutura do solo, com aumentos de densidade e resistência do solo à penetração, onde houve tráfego de máquinas na entrelinha da cultura (ARCOVERDE et al., 2019b).

Ressalta-se que os valores de densidade do solo variaram entre 1,60 e $1,82 \mathrm{~g} \mathrm{~cm}^{-3}$ nas áreas com tráfego 
de equipamentos, enquanto essa variação foi entre 1,50 e $1,78 \mathrm{~g} \mathrm{~cm}^{-3}$, nas áreas sem tráfego de equipamentos. Esses valores de densidade estão de acordo com os apresentados por Dias et al. (2016) que, estudando um latossolo de textura franco-arenosa, sob pastagem, verificaram valores de densidade entre 1,50 e $1,82 \mathrm{~g} \mathrm{~cm}^{-}$ 3. Isso evidencia a importância de se conhecer o momento adequado para retomada da colheita mecanizada, como ocorreu no quarto dia, em que se verificou menor densidade do solo, e tendo sido esse valor considerado normal para uma lavoura de cana-deaçúcar recentemente implantada (Carvalho et al., 2011). Enquanto os maiores valores de densidade do solo, em profundidade, estão de acordo com Tormena et al. (2002), que ao avaliarem o preparo mínimo, plantio direto e plantio convencional em latossolo vermelho distrófico, constataram uma densidade significativa para a camada subsuperficial nos sistemas de preparo, principalmente pelos efeitos do tráfego excessivo de máquinas agrícolas aliado à ação das ferramentas dos implementos de preparo do solo abaixo da camada arável do solo.

A porosidade total do solo obteve menores valores na profundidade $0-0,10 \mathrm{~m}$ em todos os dias avaliados (Tabela 3), ou seja, a camada superficial foi a que mais sofreu alteração da porosidade total com o tráfego de equipamentos (CTE), em relação aos dados obtidos sem o tráfego de equipamentos (STE). No quarto dia de avaliação, a camada mais profunda do solo $(0,20-0,30$ m) foi a que menos sofreu alteração da porosidade total do solo com o tráfego de equipamentos (CTE); a partir do segundo dia de avaliação, tanto a camada intermediária $(0,10-0,20 \mathrm{~m})$ como a mais profunda $(0,20-0,30 \mathrm{~m})$, sofreram menores alterações na porosidade total do solo com o tráfego de equipamentos (CTE).

Tabela 3: Porosidade total em porcentagem \%, latossolo na região de Caarapó MS.

\begin{tabular}{ccccccc}
\hline & \multicolumn{6}{c}{ Profundidade (m) } \\
\cline { 2 - 7 } Dias & \multicolumn{2}{c}{$0-0,10$} & \multicolumn{2}{c}{$0,10-0,20$} & \multicolumn{2}{c}{$0,20-0,30$} \\
\hline & CTE & STE & CTE & STE & CTE & STE \\
1 & 30,22 a B & 35,76 b A & 29,43 a & 31,90 bc & 27,97 b B & 33,47 ab A \\
2 & 29,51 a B & 36,06 b A & 29,50 a & 30,31 c & 28,77 b A & 27,62 b A \\
3 & 32,49 a B & 40,50 a A & 30,38 a & 35,91 ab & 28,36 b A & 31,47 ab A \\
4 & 32,43 a B & 42,79 a A & 34,89 a & 37,63 a & 35,10 a A & 34,26 a A \\
\hline
\end{tabular}

CTE: com tráfego de equipamentos, STE: sem tráfego de equipamentos. Letras iguais maiúsculas na linha e minúscula na coluna, não diferem entre si pelo teste Tukey 0,05 .

A correlação entre umidade, densidade e porosidade é apresentada na Tabela 4, em que se verificou correlação significativa a $1 \%$ e positiva para umidade e densidade do solo, portanto, quando houve o aumento da umidade do solo, também ocorreu aumento da densidade do solo. Segundo o trabalho de Timm et al. (2006), a umidade do solo e a porosidade do solo podem influenciar diretamente nas propriedades físicas do solo, ou seja, influenciam diretamente o processo de compactação; desse modo, solos com elevados valores de umidade ou saturados são aqueles com maior suscetibilidade à compactação devido ao tráfego de equipamentos agrícolas.

A correlação entre a densidade do solo e porosidade total do solo também foi significativa a $1 \%$, e segundo os dados obtidos a correlação entre esses dois fatores foi negativa, ou seja, quando houve o aumento da densidade do solo ocorreu a diminuição da porosidade total do solo. Como se esperava, esses dois fatores caminhariam em sentidos opostos, segundo Streck et al. (2004), isso se explica pelo fato de quando se aumenta a resistência do solo, tornando-o mais denso, isso diretamente faz com que o mesmo fique menos poroso. Enquanto não houve significância na correlação entre a umidade do solo e porosidade total do solo, o que demonstra maior sensibilidade da densidade do solo como indicador de degradação estrutural do solo como consequência das práticas de manejo, corroborando com estudos realizados por Arcoverde et al. (2019a) e Arcoverde et al. (2019b).

Tabela 4: Correlação linear de Pearson entre os dados de umidade, porosidade total e densidade do solo.

\begin{tabular}{lccc}
\hline & \multicolumn{3}{c}{ Correlação linear } \\
\cline { 2 - 4 } Avaliação & Densidade & Porosidade total & Umidade \\
\hline Densidade & 1 & & \\
Porosidade total & $-0,582^{* *}$ & 1 & 1 \\
Umidade & $0,348^{* *}$ & -0.07 & \\
\hline
\end{tabular}

*Correlação significativa, $P<0,01$.

Segundo o estudo de Callegari-Jacques (2003), sobre a categorização para os valores do coeficiente de correlação de Pearson, sugere que a categoria das correlações lineares se classifica em: nula $(0$ a $<0,1)$, fraca $(0,1$ a $<0,3)$, moderada $(0,3$ a $<0,6)$, forte $(0,6$ a $<0,9)$, muito forte $(0,9$ a $<1,0)$ e perfeita $(1,0)$.

Com base nesses dados, a correlação dos fatores umidade do solo e densidade do solo com dados obtidos de 0,348 foi classificada como moderada, e a correlação entre densidade do solo e porosidade total do solo com dados obtidos de $-0,582$, também foi classificada como moderada chegando próximo da classificação forte. A correlação da umidade do solo com a porosidade total do solo foi de -0.07 , então a mesma foi classificada como nula. 


\section{Considerações finais}

Houve maior compactação do solo com o tráfego de máquinas na retomada de colheita de cana na camada superficial em todos os dias avaliados. A densidade do solo teve menor alteração quando a retomada da colheita foi realizada 4 dias após a chuva e, consequentemente, este foi o dia em que a porosidade do solo também foi maior. A densidade do solo mostrou-se um indicador sensível à compactação como consequência do tráfego de máquinas para retomada de colheita de cana.

\section{Referências}

Arcoverde, S. N. S.; Souza, C. M. A.; Santos, W. R.; Nagahama, H. J.; Suaréz, A. H. T.; Maciak, P. A. G. (2019b). Atributos físicos de latossolo vermelho distroférrico cultivado com cana-de-açúcar em função do preparo e local de amostragem. Cultura Agronômica, 28:(4), 478-492, 2019b..

ARCOVERDE, S.N.S.; SOUZA, C.M.A.; SUAREZ, A.H.T.; COLMAN, B.A.; NAGAHAMA, H.J. Atributos físicos do solo cultivado com cana-de-açúcar em função do preparo e época de amostragem. Revista de Agricultura Neotropical, 6:(1), 41-47, 2019a.

CALLEGARI-JACQUES, S.M. Bioestatística: princípios e aplicações. Porto Alegre, RS: Artemed, 2003, p. 255.

CARVALHO, L.A.; SILVA JUNIOR, A.A.; NUNES, W.A.G.A.; MEURER, I.; SOUZA JÚNIOR, W.S. Produtividade e viabilidade econômica da cana-deaçúcar em diferentes sistemas de preparo do solo no Centro-oeste do Brasil. Revista de Ciências Agrárias, 34:(1), 200-211, 2011.

CHERUBIN, M.R.; KARLEN, D.L.; FRANCO, A.L.V.; TORMENA, C.A.; CERRI, E.P.; DAVIES, C.A.; CERRI, C.C. Soil physical quality response to sugarcane expansion in Brazil. Geoderma, 26:(1), 156$168,2016$.

DIAS, C.B.; ROCHA, G.C.; ASSIS, I.R. de; FERNANDES, R.B.A. Intervalo hídrico ótimo e densidade crítica de um Latossolo Amarelo coeso sob diferentes usos no ecossistema Tabuleiro Costeiro. Revista Ceres, 62:(6), 868-878, 2016.

FIETZ, C.R.; FISCH, G.F.; COMUNELLO, E.; FLUMIGNAN, D.L. O clima da região de Dourados, MS. Dourados: Embrapa Agropecuária Oeste. 31p. 2017.
GARCÍA, A.H.; NATERA, J.M.; VELÁZQUEZ, C.; GIL, A. Sugarcane root under regular soil water content, compaction, irrigation and shear stress. Saber, 28:(3), 480-493, 2016.

GUIMARÃES JÚNNYOR, W. DA S.; DE MARIA, I.C.; ARAUJO-JUNIOR, C.F.; LIMA, C.C. DE, VITTI, A.C.; FIGUEIREDO, G.C.; DECHEN, S.A.F. Scientia Agricola, 76:(6), 509-517, 2019.

PRADO, H. Pedologia Fácil: Aplicações em solos tropicais. Piracicaba, SP: 2013, p.284.

REINERT, D.J.; ALBUQUERQUE, J.A.; REICHERT, J.M.; AITA, C.; ANDRADA, M.M.C. Limites críticos de densidade do solo para o crescimento de raízes de plantas de cobertura em Argissolo Vermelho. Revista Brasileira de Ciência do Solo, 32:(5), 1805-1816, 2008.

SEVERIANO, E.C.; OLIVEIRA, G.C.; DIAS JUNIOR, M.S.; OLIVEIRA, L.F.C.; CASTRO, M.B. Pressão de preconsolidação e intervalo hídrico ótimo como indicadores de alterações estruturais de um Latossolo e de um Cambissolo sob cana-de-açúcar. Revista Brasileira de Ciência do Solo, 32:(4), 14191427, 2008.

SOUZA, A.C.M. de.; FARHATE, C.V.V.; SOUZA, Z.M. de.; TORRES, L.R.; SILVA R.B. da. Soil LoadBearing Capacity and Development of Root System in Area Under Sugarcane with Traffic Control in Brazil. Sugar Tech, 21:(1), 153-161, 2019.

STRECK, C.A.; REINERT, D.J.; REICHERT, J.M.; KAISER, D.R. Modificações em propriedades físicas com a compactação do solo causada pelo tráfego induzido de um trator em plantio direto. Ciência Rural, 34:(3), 755-760, 2004.

TEIXEIRA, P.C.; DONAGEMMA, G.K.; FONTANA, A.; TEIXEIRA, W.G. Manual de métodos de análise de solo. Embrapa Informação Tecnológica, Brasília, DF, 2017.

TIMM, L.C.; PIRES, L.F.; REVERATTI, R.; ARTHUR, R.C.J.; REICHARDT, K.; OLIVEIRA, J.C.M.; BACCHI, O.O.S. Field spatial and temporal patterns of soil water content and bulk density changes. Scientia agricola, 63:(1), 55-64, 2006.

TORMENA, C.A.; BARBOSA, M.C.; COSTA, A.C.S.; GONÇALVES, A.C.A. Densidade, porosidade e resistência à penetração em Latossolo vermelho distrófico sob diferentes sistemas de preparo do solo. Scientia Agricola, 59:(4), 795-801, 2002. 